\title{
Historical essays on mycology
}

C. T. Ingold

Introduction to the History of $\mathrm{Mycol}$ ogy. By G. C. Ainsworth. Pp. xi+359. (Cambridge University: Cambridge, London and New York, 1976.) $£ 11$.

Mrcologists have always lacked a comprehensive history of their subject. Many of the older mycologists have read with profit Whetzel's An Outline of the History of Phytopathology (1918) and have relished Ramsbottom's The Expanding Knowledge of Mycology Since Linnaeus (1941). And a much wider circle has also rejoiced in Large's The Advance of the Fungi. But now at last here is a full-scale history of mycology, although, with characteristic modesty, the author presents it as an Introduction.

Only one with an encyclopaedic knowledge of the literature on fungi could have produced this work which fittingly comes from the pen of one who can be described as the Dr Johnson of mycology. No other worker in this field can exist without almost daily reference to his Dictionary of Fungi.

Dr Ainsworth has sensibly not attempted to keep all sections of the subject in play while proceeding steadily from the earliest times to the present day, but has rather produced a series of historical essays on various aspects of mycology. These form his chapter headings: origin and status of fungi; form and structure; culture and nutrition; sexuality, cytology and genetics; pathogenicity; poisonous, hallucinogenic and allergenic fungi; classification; and finally the organisation of mycology.

P. A. Micheli, born in 1679 and the author of Nova Plantarum Genera (1729), is rightly regarded as starting the scientific study of the fungi; as the father of mycology. We learn from the English translation of his epitaph in Florence that he "was much beloved by all the worthy men of his age on account of his wisdom, sweetness of disposition and modesty". A number of figures from his magnum opus are reproduced: the full title-page; his lovely plate of Clathrus; his representation of agaric structure and development: his drawings of almost pure cultures of moulds on melon slices; his illustrations of Aspergillus and Botrytis; and his dynamic figure of a cup-fungus

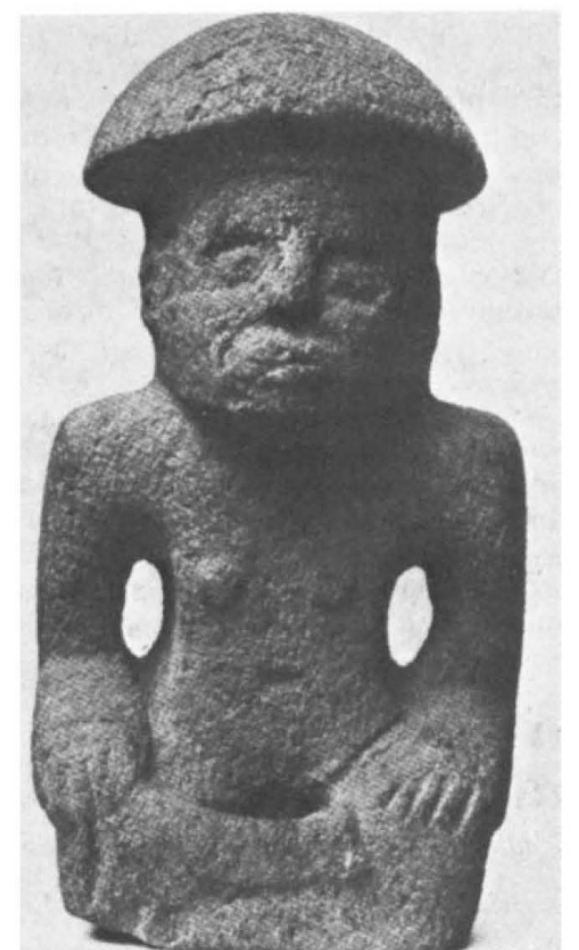

Mayan mushroom stone (British Museum, London), probably from Guatemala and believed to date from the middle of the first millenium $A D$-early evidence for a mushroom cult in Central America.

puffing. Also reproduced are Micheli's original drawings of asci, the first ever made, showing clearly that the blockmaker did a rather poor job at times in preparing the plates for the printed book.

There is a basic difficulty about a history that extends from the misty past to the present. In the earlier part much must be made from very little: and in the more recent part there is such a wealth of material that the historian is faced with an almost impossible task of selective condensation. It is, of coursc, easy to criticise this sclection from recent history. For example, in connection with mycorrhiza no mention is made of Mclin's work and the reader is referred for a recent view on ericaccous mycorrhiza to a 1927 review by Rayner! Again, in the genetics section the early work of Hansen and Smith in 1932 on heterokaryosis does not figure; indeed, the word does not occur in the index. But any criticisms of this nature are of marginal significance.

The chapter on pathogenicity is particularly rewarding because the author, with his profound knowledge of medical and veterinary mycology, presents a balanced picture in which diseases of plants and of animals (including man) are nicely balanced. Occasionally, perhaps inevitably, a chapter becomes something of a catalogue and this is most noticeable in the chapters on the distribution of fungi and on classification.

Illustrations are a special feature of the book. In all, 106 carefully selected figures-including reproductions of historic drawings; portraits and photographs of eminent mycologists; and facsimile reproductions of pages from books and manuscripts-provide a distinguished atmosphere for the book.

A brief section which many will enjoy deals with hallucinogenic fungi. The Wassons together with Roger Heim are regarded as the founders of ethnomycology.

The final chapter on the history of the organisation of the subject is stimulating. It is concerned with mycological societies, journals and books. Due credit is given to de Bary's textbook. Although over a hundred years old, this great work is still a model that has never been surpassed and is still an inspiration to working mycologists. The same chapter is enlivened by one of Worthington Smith's delightful cartoons of fungal forays a century ago.

Another mycologist writing a history (but it is difficult to think of anyone else who could do it) would certainly achieve a different balance. To some extent the balance reflects Dr Ainsworth's own interests but happily these are exceptionally wide. It is perhaps not a book for the undergraduate student, but it will be read with enormous pleasure by all mycologists with a broad interest in their subject. Every library in an institution where fungi are studied will have to possess a copy and many individual workers will want one of their own.

C. T. Ingold was Professor of Botany at Birkbeck College, University of London, UK, from 1944 to 1972. 